\title{
Research on Fire Risk Safety Evaluation of Tank Farm based on Improved Fuzzy AHP Method
}

Qilei Wang, Benli Li, Xifeng Kang, Fang Zhang, Chunlei Jia, Xiaoming Zhang

China People's Police University

Hebei Langfang, China 403115051@qq.com

基于改进模糊层次分析法的油罐区火灾 风险安全评价研究

王其䂞, 李本利, 康习锋, 张芳, 贾春雷, 张晓明 中国人民警察大学

河北 廊坊 065000 中国

\section{1@qq.com}

Abstract-From the fire safety point of view, the impact factors and evaluation index of fire safety in tank farm are important to fire safety and fire rescue system. The tank farm fire risk assessment system was structured by multi coupling relationship of fire safety was integrated used and improved fuzzy AHP method was used in the fire safety evaluation, fire hydrant system, nitrogen cooling and evacuation system. Using expert scoring method to evaluate the index weight, and the original fuzzy analysis hierarchy model was improved. The results show that: the method reduces the weight of the evaluation subjectivity, and evaluate the overall effect is better, the results show that the model is reasonable and feasible. The improved fuzzy AHP evaluation method applied to fire risk evaluation of tank farm is practical and effective.

Keywords- fire risk; tank farm; improved fuzzy AHP method; safety evaluation

摘要一消防安全性角度对油罐区中火灾风险的影 响因素及评价指标进行分析, 对消防安全及灭火救 援保障具有重要的意义。综合运用消防安全多元耦 合关系及改进模糊层次分析法对影响消防安全的 报警系统、消火栓给水系统、氮冷却系统、疏散系 统、消防队救援等的重要性及合理程度进行深入分 析, 构建了油罐区火灾风险消防安全评价体系。运 用专家打分法对指标权重份数进行了评价, 并对
原有的模糊分析层次模型进行改进，对某油罐区火 灾风险进行实例验证。结果表明：该方法减少了确 定权重的主观性，评价总体效果较好，结果表明该 模型合理、可行，改进模糊层次评价方法应用于油 罐区火灾风险消防安全评价是实用且有效的。

关键词一火灾风险，油罐区，改进的模糊分析层 次模型，安全评价

I. 引 言

随着我国石油化工产业的持续发展以及 国家石油战略储备制度的建立, 石油的储量必 将越来越大。油罐内储存的石油及其产品均为 易燃、易爆物质，极易导致的火灾、爆炸事故 的发生, 储罐大型化的发展也给火灾预防和扑 救带来了新的挑战, 油罐火灾一旦发生, 扑救 难度很大, 在扑救过程中需要使用大量的水配 制泡沫混合液进行着火储罐和地面流淌火的 扑救。因此有效的降低油罐区火灾风险, 对油 罐区火灾风险进行安全评价显得至关重要。目 前, 国内外相关研究明确了油罐火灾必须立足 于自救与外部救援，即依托技术工人的关阀断 料与固定消防设施进行灭火救援和疏散, 又要 依托外部消防救援力量 ${ }^{[1-3]}$ 。

结合实际情况，如何评价油罐区火灾风险 安全指数对于整个油罐区安全系统起着至关 重要的作用。对于油罐区火灾风险安全性评价 主要采用层次分析法, 它是一种多层次权重分 析决策方法, 但是在进行判断矩阵标准修正 
时, 主观性强, 缺乏科学的理论和方法。国外 消防相关部门主要通过严格遵守消防设计规 范, 提高油罐区安全设计规范, 加强固定消防 设施可靠性能, 消防队制定严格的灭火作战预 案等方法提高消防安全可靠性, 降低油罐区 火灾风险指数。

本文主要依据相关国家标准规范、专家经 验及改进模糊层次分析评价方法, 对油罐区安 全分布结构、工人技术水平、消火栓给水系统、 自动冷却供水系统的给水方式和消防队伍等 相关影响因素权重及评价办法进行关联分析, 对降低油罐区火灾风险具有重要的现实意义。

\section{П. 评价体系}

油罐区消防安全评价指标体系是评价油 罐区火灾风险指数的基础。选择评价指标不仅 要考虑油罐区本身及消防设施状态, 而且要反 映出对消防安全有潜在影响的重要因素的变 化、人类活动的影响以及指标数据的可获得 性。本文主要依据相关学者在安全评价研究方 面的成果，结合我国油罐区消防安全的实际 特征, 利用客观性与易操作性原则, 根据评价 指标相关性原则，建立多层因素集。在综合分 析与调查研究的基础上，提出了油罐区消防 安全评价体系，如表1所示。

表1 油罐区消防安全评价指标体系

\begin{tabular}{|c|c|c|c|}
\hline 第一层 & 第二层 & 第三层 & 第四层 \\
\hline \multirow{20}{*}{$\begin{array}{l}\text { 油罐 区火灾 } \\
\text { 安全(A) }\end{array}$} & \multirow{6}{*}{$\begin{array}{l}\text { 主动防火 } \\
\text { (B1) }\end{array}$} & \multirow{4}{*}{ 消防系统(C1) } & 消防探测与报警系统C11 \\
\hline & & & 自动喷水系统C12 \\
\hline & & & 氮冷却系统C13 \\
\hline & & & 消防栓系统C14 \\
\hline & & \multirow{3}{*}{ 消防队(C2) } & 最近消防队响应时间 $\mathrm{C} 21$ \\
\hline & & & 消防队灭火救援能力 $\mathrm{C} 22$ \\
\hline & \multirow{8}{*}{$\begin{array}{l}\text { 被动防火 } \\
\text { (B2) }\end{array}$} & & 防火间距与火灾载荷 $\mathrm{C} 31$ \\
\hline & & \multirow{4}{*}{$\begin{array}{l}\text { 油罐区结构设计 } \\
\text { (C3) }\end{array}$} & 房屋建筑结构C32 \\
\hline & & & 防火分隔C33 \\
\hline & & & 消防疏散与安全出口C34 \\
\hline & & & 建筑构件耐火性能 C41 \\
\hline & & \multirow{3}{*}{$\begin{array}{l}\text { 油罐区安全阻燃 } \\
\text { (C4) }\end{array}$} & 装修材料耐火性能C42 \\
\hline & & & 公共物品耐火性能C43 \\
\hline & & & 建筑物电路老化程度 C44 \\
\hline & \multirow{6}{*}{$\begin{array}{l}\text { 消防安全管理 } \\
\text { (B3) }\end{array}$} & \multirow{4}{*}{$\begin{array}{l}\text { 油罐 区内部人员 } \\
\text { (C5) }\end{array}$} & 人员密度C51 \\
\hline & & & 年龄组成C52 \\
\hline & & & 消防员素质情况C53 \\
\hline & & & 消防管理规章C61 \\
\hline & & \multirow[t]{2}{*}{ 管理水平(C6) } & 技术工人C62 \\
\hline & & & 专业消防力量C63 \\
\hline
\end{tabular}

Ш. 改进模糊层次分析法

改进的模糊层次分析法的主要特点之一 是模糊一致性矩阵, 该矩阵能够满足一致性条 件, 无需进行一致性检验, 不仅能减少迭代次 数, 而且比传统的优先判断矩阵的收玫速度更 快, 同时又能够满足计算精度的要求。

(1) 建立优先判断矩阵 $F=\left\{f_{i j}\right\}_{n \times n}$, 该矩阵是 模糊互补矩阵, 矩阵中的值采用两元素标度 法, 选用0.1 0.9标度表示, 该方法能够准确地
表2 元素标度

\begin{tabular}{lll}
\hline 标度 & 比较 & 定义 \\
\hline 0.5 & 同等重要 & 两个因素相比, 具有相同的重要性 \\
0.6 & 稍微重要 & 两个因素相比, 前者比后者稍微重要 \\
0.7 & 明显重要 & 两个因素相比, 前者比后者明显重要 \\
0.8 & 重要得多 & 两个因素相比, 前者比后者重要的多 \\
0.9 & 极端重要 & 两个因素相比, 前者比后者极端重要 \\
$0.1,0.2$ 反比较 & 若元素 $a_{i}$ 与元素 $a_{j}$ 相比较得到判断 $f_{i j}$, \\
$0.3,0.4$ & 则元素与元素相比较得到的判断为 $f_{j i}=1-f_{i j}$ \\
\hline
\end{tabular}


描述任意两个因素关于某准则的相对重要程

度, 本文标度法确定的元素值, 如表2所示。

(2) 求行和 $r_{i}=\sum_{j=1}^{n} f_{i j}$, 并利用转换公式 $r_{i}=\frac{r_{i}-r_{j}}{2 n}+0.5$, 将模糊判断矩阵 $F=\left\{f_{i j}\right\}_{n \times n}$ 改造成模糊一致性判断矩阵 $R=\left\{r_{i j}\right\}_{n \times n}$.

(3) 方根法求排序向量 $\mathrm{W}^{(0)}$ :

$$
\begin{aligned}
& W^{(0)}=\left\{\begin{array}{llll}
\omega_{1} & \omega_{2} & \ldots & \omega_{\mathrm{n}}
\end{array}\right\}^{T}= \\
& {\left[\frac{\sqrt[n]{\prod_{i=1}^{n} e_{1 j}}}{\sum_{i=1}^{n} \sqrt[n]{\prod_{j=1}^{n} e_{i j}}} \frac{\sqrt[n]{\prod_{i=1}^{n} e_{2 j}}}{\sum_{i=1}^{n} \sqrt[n]{\prod_{j=1}^{n} e_{i j}}} \ldots \frac{\sqrt[n]{\prod_{i=1}^{n} e_{n j}}}{\sum_{i=1}^{n} \sqrt[n]{\prod_{j=1}^{n} e i j}}\right]^{T}}
\end{aligned}
$$

(4)利用转换公式 $e_{i j}=\frac{r_{i j}}{r_{j i}}$ 将互补型判断矩阵 $R=\left\{r_{i j}\right\}_{n \times n}$ 变为互反型矩阵 $E=\left\{e_{i j}\right\}_{n \times n}$.

(5) 以排序向量 $\mathrm{W}^{(0)}$ 作为特征值法的迭代出 值法的迭代初值 $\mathrm{V}_{0}$, 求精度较高的排序向量 $\mathrm{W}^{(\mathrm{k})}$

$V_{0}=V_{0}\left(\begin{array}{llll}\omega_{1} & \omega_{2} & \cdots & \omega_{n}\end{array}\right)^{\mathrm{T}}$ 为迭代初值 $\mathrm{V}_{0}$ 值, 利用迭代公式 $V_{k+1}=E V_{k}$ 求特征向量 $V_{k+1}$, 并求 $\mathrm{V}_{\mathrm{k}+1}$ 的无穷范数 $\left\|V_{k+1}\right\|_{\infty}$ 。

判断: 若 $\left\|V_{k+1}\right\|_{\infty}-\left\|V_{k}\right\| \leq \varepsilon$, 则 $\left\|V_{k+1}\right\|_{\infty}$ 即为最 大特征值 $\lambda_{\text {max }}$ ，将 $\mathrm{V}_{\mathrm{k}+1}$ 进行归一化处理：

$$
V_{k+1}=\left[\begin{array}{llll}
\frac{v_{k+1,1}}{\sum_{i=1}^{n} v_{k+1, i}} & \frac{v_{k+1,2}}{\sum_{i=1}^{n} v_{k+1, i}} & \cdots & \frac{v_{k+1, n}}{\sum_{i=1}^{n} v_{k+1, i}}
\end{array}\right]^{T}
$$

所得向量 $\mathrm{W}^{(\mathrm{k})}=\mathrm{V}_{i+1}$ 即为方案排序向量, 迭代 结束.

否则, 以 $V_{k+1}=\frac{V_{k+1}}{\|V k+1\|_{\infty}}=\left[\begin{array}{llll}\frac{v_{k+1,1}}{\left\|V_{k+1}\right\|_{\infty}} & \frac{v_{k+1,2}}{\left\|V_{k+1}\right\|_{\infty}} & \cdots & \frac{v_{k+1, n}}{\left\|V_{K+1}\right\|_{\infty}}\end{array}\right]^{T}$
作为新初值, 再次迭代。

IV. 指标权重与因素集的选择

1 评估指标

油罐区消防安全评估指标集的选取方法 通常参照油罐区安全标准, 按照消防设施可靠 度和建筑设计规范, 综合消防工程的实际情 况, 通常将油罐区的安全性(火灾风险)划分成 以下 4 个等级：安全、较安全、不安全、很不 安全, 见表 3:

表 3 油罐区消防安全等级

安全等级 安全 较安全 不安全 很不 安全

\begin{tabular}{l}
\hline 评价值 $0.9 \sim 1 \quad 0.7 \sim 0.9$ \\
$<0.5$ \\
\hline 2 油罐区安全评价结果 \\
根据所得对油罐区消防安全评价指标的
\end{tabular}
组合权重进行分析, 结合消防安全的现场调查 结果与专家评分法, 得到安全评价指标体系中 各指标的评价值 $\left(F_{i}\right)$, 并采用 $Q=\sum_{i=1}^{n} F_{i} N_{i} / 100$, 其中: $\mathrm{Q}$ 为最终安全评价值; $F_{i}$ 为评价指标评 价值; $N_{i}$ 为指标组合权重。依据油罐区消防安 全等级设定, 对该油罐进行火灾风险等级划 分。

$$
\text { V. 应用实例 }
$$

1 建筑分析实例

某油罐区, 罐区内共有四个储罐, 储存介 质为柴油和汽油, 储罐区长度 $129 \mathrm{~m}$, 储罐长 款为 $60 \mathrm{~m}$, 罐体间距为 $9 \mathrm{~m}$, 其中 1,2 号罐为内 浮顶, 储罐体积 $10000 \mathrm{~m}^{3}$, 油品体积 $8500 \mathrm{~m}^{3}, 3$, 4 号罐为拱顶 $10000 \mathrm{~m}^{3}$, 油品体积 $8000 \mathrm{~m}^{3}$, 耐火 等级为一级。

根据该油罐区消防安全调查结果, 依据专 家打分法, 得出各安全评价指标的评价值, 如 表4所示。

表 4 消防安全评价指标值

\begin{tabular}{cccc}
\hline 评价指标 & 评价值 & 评价指标 & 评价值 \\
$\mathrm{C} 11$ & 85 & $\mathrm{C} 41$ & 76 \\
$\mathrm{C} 12$ & 87 & $\mathrm{C} 42$ & 79 \\
$\mathrm{C} 13$ & 83 & $\mathrm{C} 43$ & 75 \\
$\mathrm{C} 14$ & 85 & $\mathrm{C} 44$ & 85 \\
$\mathrm{C} 21$ & 80 & $\mathrm{C} 51$ & 78 \\
$\mathrm{C} 22$ & 82 & $\mathrm{C} 52$ & 80 \\
\hline
\end{tabular}




\begin{tabular}{llll}
\hline $\mathrm{C} 31$ & 83 & $\mathrm{C} 53$ & 75 \\
$\mathrm{C} 32$ & 79 & $\mathrm{C} 61$ & 75 \\
$\mathrm{C} 33$ & 83 & $\mathrm{C} 62$ & 83 \\
$\mathrm{C} 34$ & 81 & $\mathrm{C} 63$ & 76 \\
\hline
\end{tabular}

2 改进的模糊层次分析法计算权重

依据表1对评价指标两两相对重要性的判 断, 并结合表2对该油罐区指标结构体系4个层 次 10 个优先判断矩阵分别为:

A-B优先判断矩阵: $\left[\begin{array}{ccc}0.5 & 0.6 & 0.7 \\ 0.4 & 0.5 & 0.6 \\ 0.3 & 0.4 & 0.5\end{array}\right]$

$\mathrm{B}_{1}-\mathrm{C}$ 优先判断矩阵: $\left[\begin{array}{ll}0.5 & 0.6 \\ 0.4 & 0.5\end{array}\right]$

B2-C优先判断矩阵 $\left[\begin{array}{ll}0.5 & 0.6 \\ 0.4 & 0.5\end{array}\right]$

B3-C优先判断矩阵 $\left[\begin{array}{ll}0.5 & 0.7 \\ 0.3 & 0.5\end{array}\right]$

$\mathrm{C}_{1}-\mathrm{C}_{1 i}$ 优先判断矩阵: $\left[\begin{array}{cccc}0.5 & 0.6 & 0.7 & 0.7 \\ 0.4 & 0.5 & 0.6 & 0.7 \\ 0.3 & 0.4 & 0.5 & 0.6 \\ 0.3 & 0.3 & 0.4 & 0.5\end{array}\right]$

$\mathrm{C}_{2}-\mathrm{C}_{2 i}$ 优先判断矩阵: $\left[\begin{array}{ll}0.5 & 0.6 \\ 0.4 & 0.5\end{array}\right]$

$\mathrm{C}_{3}-\mathrm{C}_{3 i}$ 优先判断矩阵: $\left[\begin{array}{cccc}0.5 & 0.7 & 0.7 & 0.8 \\ 0.3 & 0.5 & 0.6 & 0.7 \\ 0.3 & 0.4 & 0.5 & 0.6 \\ 0.2 & 0.3 & 0.4 & 0.5\end{array}\right]$

$\mathrm{C}_{4}-\mathrm{C}_{4 i}$ 优先判断矩阵: $\left[\begin{array}{cccc}0.5 & 0.6 & 0.6 & 0.7 \\ 0.4 & 0.5 & 0.6 & 0.6 \\ 0.4 & 0.4 & 0.5 & 0.7 \\ 0.3 & 0.4 & 0.3 & 0.5\end{array}\right]$

$\mathrm{C}_{5}-\mathrm{C}_{5 i}$ 优先判断矩阵: $\left[\begin{array}{ccc}0.5 & 0.6 & 0.6 \\ 0.4 & 0.5 & 0.6 \\ 0.4 & 0.4 & 0.5\end{array}\right]$
$\mathrm{C}_{6}-\mathrm{C}_{6 i}$ 优先判断矩阵: $\left[\begin{array}{ccc}0.5 & 0.6 & 0.8 \\ 0.4 & 0.5 & 0.6 \\ 0.2 & 0.4 & 0.5\end{array}\right]$

以 $\mathrm{C}_{4}-\mathrm{C}_{4 i}$ 优先判断矩阵为例进行计算, 利用转 换公式, 得到模糊一致性判断矩阵:

$$
R=\left\{r_{i j}\right\}_{5 \times 5}=\left[\begin{array}{cccc}
0.50 & 0.55 & 0.59 & 0.66 \\
0.45 & 0.50 & 0.58 & 0.63 \\
0.41 & 0.42 & 0.50 & 0.56 \\
0.34 & 0.37 & 0.44 & 0.50
\end{array}\right]
$$

方根法求排序向量

$$
W^{(0)}=\left(\begin{array}{llll}
0.237 & 0.229 & 0.189 & 0.165
\end{array}\right)
$$

通过转换公式将判断矩 $R=\left\{r_{i j}\right\}_{4 \times 4}$ 转换成互反 型矩阵 $\mathrm{E}$ :

$E=\left\{e_{i j}\right\}_{4 \times 4}=\left[\begin{array}{cccc}1 & 1.235 & 1.356 & 1.823 \\ 0.812 & 1 & 1.302 & 1.743 \\ 0.756 & 0.812 & 1 & 1.295 \\ 0.435 & 0.673 & 0.785 & 1\end{array}\right]$ 迭代计算精度 $\varepsilon=0.0001$, 令 $\mathrm{V}_{0}=\mathrm{W}^{(0)}=(0.237$, $0.229,0.189,0.165)^{\mathrm{T}}$ 作为特征值法的迭代初 值, 迭代3次后求得 $\mathrm{W}^{(3)}=(0.312,0.287,0.212$, $0.189)^{\mathrm{T}}$, 计算精度 $\left\|x_{3}\right\|_{\infty}-\left\|x_{2}\right\|_{\infty} \leq 0.0001$. 同样 的方法得到 $\mathrm{A}-\mathrm{B} 、 \mathrm{~B}_{1}-\mathrm{C} 、 \mathrm{~B}_{2}-\mathrm{C} 、 \mathrm{~B}_{3}-\mathrm{C} 、 \mathrm{C}_{1}-\mathrm{C}_{1 i}$ 、 $\mathrm{C}_{2}-\mathrm{C}_{2 i} 、 \mathrm{C}_{3}-\mathrm{C}_{3 i} 、 \mathrm{C}_{5}-\mathrm{C}_{5 i} 、 \mathrm{C}_{6}-\mathrm{C}_{6 i}$ 矩阵的结果分别 为( $\left.\begin{array}{lll}0.286 & 0.313 & 0.401\end{array}\right),\left(\begin{array}{lll}0.4 & 0.6\end{array}\right),\left(\begin{array}{ll}0.35 & 0.65\end{array}\right)$, (0.45 0.55) (0.307 0.296 0.209 0.188), (0.4 0.6), ( $0.3320 .305,0.2030 .156),(0.2960 .3430 .361)$, (0.318 0.2470 .435$)$

利用改进的模糊层次分析法模型, 结合专 家打分法及判断矩阵, 得出油罐区消防安全评 价指标体系权重, 如表5所示。

表5 改进模糊层次分析法权重值

\begin{tabular}{lllll}
\hline 优先判 & \multicolumn{4}{c}{ 权重排序 } \\
\cline { 2 - 5 } 断矩阵 & $\mathrm{W} 1$ & $\mathrm{~W} 2$ & $\mathrm{~W} 3$ & $\mathrm{~W} 4$ \\
\hline $\mathrm{A}$ & 0.286 & 0.313 & 0.401 & \\
$\mathrm{~B} 1$ & 0.4 & 0.6 & & \\
$\mathrm{~B} 2$ & 0.35 & 0.65 & & \\
$\mathrm{~B} 3$ & 0.45 & 0.55 & & \\
$\mathrm{C} 1$ & 0.307 & 0.296 & 0.209 & \\
\hline
\end{tabular}




\begin{tabular}{lllll}
\hline 0.188 & & & & \\
$\mathrm{C} 2$ & 0.4 & 0.6 & & \\
$\mathrm{C} 3$ & 0.332 & 0.305 & 0.203 & 0.162 \\
$\mathrm{C} 4$ & 0.312 & 0.287 & 0.212 & 0.189 \\
$\mathrm{C} 5$ & 0.296 & 0.343 & 0.361 & \\
$\mathrm{C} 6$ & 0.318 & 0.247 & 0.435 & \\
\hline
\end{tabular}

然后结合消防安全评价指标值与权重比, 计算 出该油罐区的 20 个安全评价指标的组合权重

$(\mathrm{Ni})$ ，见表6

\section{表6 评价指标权重值}

\begin{tabular}{llll}
\hline 评价指标 & $\mathrm{N}_{i}$ & $\mathrm{~F}_{i}$ & $\mathrm{~F}_{i} \mathrm{~N}_{i}$ \\
\hline 消防探测与报警系统 & 0.0733 & 85 & 6.231 \\
自动喷水系统 & 0.0839 & 87 & 7.299 \\
氮冷却系统 & 0.0627 & 83 & 5.204 \\
消防栓系统 & 0.0632 & 85 & 5.372 \\
最近消防队响应时间 & 0.0536 & 80 & 4.288 \\
消防队灭火救援能力 & 0.0543 & 82 & 4.453 \\
防火间距与火灾载荷 & 0.0492 & 83 & 4.084 \\
房屋建筑结构 & 0.0503 & 79 & 3.974 \\
防火分隔 & 0.0519 & 83 & 4.308 \\
消防疏散与安全出口 & 0.0486 & 81 & 3.937 \\
建筑构件耐火性能 & 0.0498 & 76 & 3.785 \\
装修材料耐火性能 & 0.0593 & 79 & 4.685 \\
公共物品耐火性能 & 0.0497 & 75 & 3.726 \\
电路老化与防爆程度 & 0.0494 & 85 & 4.199 \\
人员密度 & 0.0283 & 78 & 2.207 \\
年龄组成 & 0.0297 & 80 & 2.376 \\
消防员素质情况 & 0.0291 & 75 & 2.183 \\
消防管理规章 & 0.0381 & 75 & 2.856 \\
技术工人力量 & 0.0379 & 83 & 3.146 \\
专业消防力量 & 0.0432 & 76 & 3.283 \\
\hline 计公式
\end{tabular}

合计算公式, 由表中数值计算:

$$
Q=\sum_{i=1}^{n} F_{i} N_{i} / 100=0.775
$$

由计算结果可以得出, 该油罐区消防安全 最终评价分数为 0.775 。对照表 3 可以发现：该 油罐区属于较安全的状态, 火灾风险较低。并
且比较表5得出: 在油罐区的消防安全评价体 系中报警系统与冷却供水等系统对消防的安 全与救援影响最大, 这与油罐区自身防火及火 灾初期安全稳定灭火性能较为重要, 并且防火 间隔与建筑材料等也是消防安全的重要影响 因素。

\section{VI. 结论}

(1) 油罐区火灾风险高低与内部消防设施 安全及可靠性有重要关系, 有效分清不同部分 权重及相互耦合关系对于油罐区消防安全至 关重要。在综合分析与调查研究的基础上, 给 出了油罐区消防安全 (火灾风险) 评价体系, 建 立了改进模糊层次分析模型, 并对指标权重与 因素集进行选择。

(2) 通过例举实例, 分析了不同影响因素 的重要程度及权重比, 并对该油罐区进行了计 算分析, 研究发现该方法能够较好的对油罐区 的火灾风险影响因素及安全程度进行有效评 价, 并且确立了消防固定设施的重要程度, 从 而进一步明确了油罐区超立足于防火自救这 一概念。

\section{致谢}

本项目受河北省重点研发计划项目 “基 于远程供水系统技术性能的油罐火灾扑救供 水力量编成研究” (编号: 17215604) 资助。

\section{参考文献}

[1] 刘金玉, 王殿生, 胡玉琴.基于层次分析法的油库区静 电风险模糊综合评价方法[J].西安石油大学学报, vol.34, issue 1, pp. 120-125, 2019.

[2] 周靖轩. 石油储罐区消防系统有效性评价方法[J]．消 防科学与技术, vol.33, issue 7, pp. 836-838, 2014.

[3]. Milan Mrkalj. Demonstrative implications of a new logical aggregation paradigm on a classical fuzzy evaluation model of 《Green》 buildings[J]. Communications in computer and information science, vol 44, issue3,pp.20-27, 201 